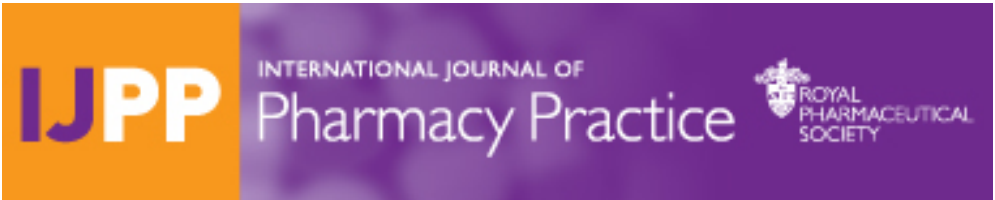

\title{
Analysis of pharmacist-identified medication-related problems at two United Kingdom hospitals: a prospective observational study
}

\begin{tabular}{|c|c|}
\hline Journal: & International Journal of Pharmacy Practice \\
\hline Manuscript ID & IJPP-19-0142.R3 \\
\hline Wiley - Manuscript type: & Research Paper \\
\hline Keywords: & $\begin{array}{l}\text { Clinical Pharmacy < Clinical Practice, Secondary Care < Delivery of Care, } \\
\text { Medication Review < Drug Utilisation, Pharmaceutical Care < Medicines } \\
\text { Management, Medication Risk < Patient Safety, Observation < Research } \\
\text { Method }\end{array}$ \\
\hline Abstract: & $\begin{array}{l}\text { Objective: Hospital pharmacy is undergoing a period of rapid change, } \\
\text { with pharmacists needing to focus where they add most value. Our aim } \\
\text { was to identify where pharmacists have potential for greatest impact by } \\
\text { analysing data on clinically relevant medication-related problems (MRPs). } \\
\text { Methods: We included consecutive admissions from adult medical wards } \\
\text { at two UK hospitals between April and November } 2016 \text {. MRPs were } \\
\text { identified by pharmacists at the study sites as part of their routine daily } \\
\text { patient assessments, validated and assessed for preventability and } \\
\text { severity. Descriptive analyses were performed on clinically relevant } \\
\text { (moderate or severe preventable) MRPs to establish the stage of } \\
\text { inpatient stay where identified and their types/categories (overall and by } \\
\text { stage of inpatient stay). } \\
\text { Key findings: Among } 1,503 \text { eligible admissions, } 2,614 \text { validated MRPs } \\
\text { were identified, of which } 1,153 \text { were moderate or severe, and } \\
\text { preventable. Over } 70 \% \text { of these clinically relevant MRPs were identified } \\
\text { during/before the first ward-based pharmacy review of patients. The } \\
\text { most frequent MRP subcategory was 'indication not treated/missing } \\
\text { therapy', accounting for } 46 \% \text { of clinically relevant MRPs. Dose selection } \\
\text { issues were the next most common, accounting for } 24 \% \text {. The } \\
\text { subcategory 'indication not treated/missing therapy' was identified more } \\
\text { frequently at admission and discharge (53\% and } 45 \% \text { of MRPs } \\
\text { respectively) compared with during the inpatient stay (14\%), p<0.001. } \\
\text { Conclusions: This research suggests patients are at greatest need of } \\
\text { pharmacist input in terms of identification/resolution of clinically relevant } \\
\text { MRPs during early stages of inpatient stay; however clinically relevant } \\
\text { MRPs continue to occur throughout their stay, suggesting need for on- } \\
\text { going pharmacy review. }\end{array}$ \\
\hline
\end{tabular}




\section{Analysis of pharmacist-identified medication-related problems at}

2 two United Kingdom hospitals: a prospective observational study

\section{ABSTRACT}

4 Objective: Hospital pharmacy is undergoing a period of rapid change, with pharmacists

5 needing to focus where they add most value. Our aim was to identify where pharmacists

6 have potential for greatest impact by analysing data on clinically relevant medication-related

7 problems (MRPs).

8 Methods: We included consecutive admissions from adult medical wards at two UK

9 hospitals between April and November 2016. MRPs were identified by pharmacists at the

10 study sites as part of their routine daily patient assessments, validated and assessed for preventability and severity. Descriptive analyses were performed on clinically relevant (moderate or severe preventable) MRPs to establish the stage of inpatient stay where identified and their types/categories (overall and by stage of inpatient stay).

Key findings: Among 1,503 eligible admissions, 2,614 validated MRPs were identified, of which 1,153 were moderate or severe, and preventable. Over $70 \%$ of these clinically relevant MRPs were identified during/before the first ward-based pharmacy review of patients. The most frequent MRP subcategory was 'indication not treated/missing therapy', accounting for $46 \%$ of clinically relevant MRPs. Dose selection issues were the next most common, accounting for $24 \%$. The subcategory 'indication not treated/missing therapy' was identified more frequently at admission and discharge ( $53 \%$ and $45 \%$ of MRPs respectively) compared with during the inpatient stay $(14 \%), p<0.001$.

Conclusions: This research suggests patients are at greatest need of pharmacist input in terms of identification/resolution of clinically relevant MRPs during early stages of inpatient stay; however clinically relevant MRPs continue to occur throughout their stay, suggesting need for on-going pharmacy review. 


\section{INTRODUCTION}

2 Hospital pharmacy in England is undergoing a period of rapid change,${ }^{1}$ driven in part by publication of Lord Carter's review of productivity in NHS hospitals; ${ }^{2}$ pharmacists are being encouraged to integrate into multidisciplinary teams and share their expertise on medicines, thereby supporting medicines optimisation and clinical care. ${ }^{13}$ Given growing demands on services, this requires improved productivity and efficiency, and a need for pharmacists to focus on 'where they are effective and add value'. ${ }^{1}$ Similarly, the NHS Long Term Plan, published in 2019, recognises the value and success of the NHS, while acknowledging concerns around funding and pressures from an ageing population; ${ }^{4}$ it sets out the NHS strategy to ensure that 'services are fit for the future', which for pharmacy requires increased focus on providing clinical services to patients. Medicines optimisation, which can be described as the safe and effective use of medicines to enable the best possible outcomes, ${ }^{3}$ is therefore a high priority for hospital pharmacy services.

In 2015 the English National Institute for Health and Care Excellence (NICE) published guidance on medicines optimisation. ${ }^{3}$ This advises that medication safety is an important consideration when optimising medicines, and highlights the considerable burden of adverse drug events. NICE estimate that errors or unintentional changes to medicines occur in $30-$ $70 \%$ of patients when they move between care settings, for example at hospital admission or discharge. ${ }^{3}$ As a result, they highlight the importance of medicines reconciliation, defined as 'the process of identifying an accurate list of a person's current medicines and comparing them with the current list in use, recognising any discrepancies, and documenting any changes'. ${ }^{3}$ NICE also recommend use of structured medication reviews for key groups of people in hospitals and primary care, for example individuals taking multiple medicines and those with chronic/long-term conditions; this includes the need to optimise the impact of medicines and minimise the number of medication-related problems (MRPs).

This need for improved medication safety is not confined to UK hospitals, ${ }^{5-8}$ resulting in international calls for improvement, such as the World Health Organization's Global Patient Safety Challenge. ${ }^{9}$ There are also international calls for increased efficiency. ${ }^{10-12}$ Research to identify hospital inpatients at greatest risk of adverse medication-related outcomes has been conducted, ${ }^{13-25}$ but research to establish the stage during hospital stay when patients may be at greatest risk of harm is limited. There is evidence that prescribing errors, a subset of MRPs, are more likely to be identified at hospital admission compared with other times during hospital stay. ${ }^{26-28}$ However, given that approximately half of all prescribing errors and MRPs that occur in hospital inpatients are of limited clinical significance, ${ }^{726}$ an understanding of the admission stage when clinically relevant MRPs are most likely to occur 
1 has potential to permit pharmacists to target patients at the stage(s) of hospital stay where

2 risk of medication-related harm is greatest. Similarly, an understanding of the

3 type/categories of clinical relevant MRPs, and the stage of hospital stay when these occur,

4 may provide increased insight into the types of intervention required.

5 The aim of this study was to identify where pharmacists have potential for greatest impact by 6 analysing data on clinically relevant medication-related problems (MRPs). This was to 7 address two gaps in the current evidence base: when clinically relevant MRPs occur in terms 8 of the stage of hospital stay, and an analysis of the types/categories of clinically relevant 9 MRPs, both overall and by stage of hospital stay. It is anticipated this may inform service delivery, with potential to improve targeting of patients requiring pharmacy input.

\section{METHOD}

\section{Study design and patients}

13 This prospective study, using an observational study design, involved patients admitted to 30 adult medical wards at two hospitals in South East England. This has been described in detail elsewhere..$^{1329} 30$ In summary, the study sites were acute district general hospitals, each with approximately 600 inpatient beds, and broadly representative of other general (non-specialist) acute NHS trusts in England. ${ }^{30}$ We included patients admitted to the general, acute, and elderly medicine wards at the study sites. Patients admitted to other specialities such as surgery, maternity and paediatrics were excluded due to potential differences in the prevalence/type of MRPs in these patient groups. At Hospital A there were 11 study wards (six general, one acute, and four elderly medicine). Hospital B had 19 study wards (six general, four acute, and nine elderly medicine). The median length of stay at both study sites was five days. Hospital A has electronic medical and prescribing records; Hospital $B$ has paper-based systems. Study wards received daily clinical pharmacy visits (Monday to Friday 9am-5pm). Medicines reconciliation routinely occurred at hospital admission, with discrepancies also identified/resolved at discharge (Hospital A and B) and when paper medication charts were rewritten (Hospital B only). A clinical pharmacy service was also available from the centralised dispensaries (Monday to Friday 9am-6.30pm and Saturday and Sunday $10 \mathrm{am}-4 \mathrm{pm})$. A sample size of 1,500 participants was selected a priori based on practical considerations. ${ }^{13}$ Eligible patients were consecutively included between April and November 2016. 
1 Ethical approval

2 This study received ethical approval in January 2016 from the Proportionate Review Service

3 Sub-Committee of the National Health Service (NHS) Research Ethics Committee Wales

4 REC 7 (16/WA/0016).

\section{Data collection}

6 As reported elsewhere, ${ }^{29}$ MRPs were defined as 'all circumstances involving a patient's drug

7 treatment that actually, or potentially, interfere with the achievement of an optimal

8 outcome'. ${ }^{31}$ We chose to study MRPs that were at least moderate in severity to inform

9 targeting of patients at highest risk of medication-related harm. Similarly, preventable MRPs

10 were studied to permit a focus on patients at risk of avoidable harm.

Following face-to-face training covering study design/purpose and MRP data collection methods, pharmacists at the study sites identified and recorded MRP data as part of their routine daily clinical assessment of patients; this included MRPs originating in both primary and secondary care. A data collection form was designed/piloted for this purpose. Data were collected during daily ward visits (Monday to Friday 9am-5pm), and by staff in the centralised pharmacy dispensaries (Monday to Friday 9am-6.30pm and Saturday and Sunday 10am$4 \mathrm{pm})$. The majority of pharmacy assessments occurred at ward level at both study sites, but data were also collected in the centralised dispensaries to permit recording of MRPs identified outside routine ward pharmacy visits, for example concerning medication requests made prior to the first ward review by pharmacy. Data collection included whether MRPs were considered preventable, (expressed as a dichotomous variable of yes or no), and the MRP type/category (see below). The following data were also recorded by pharmacy staff: (1) stage during patient stay when MRP identified, classified as during/before first ward review by pharmacist, during the remainder of the inpatient stay, or during clinical screening at discharge; and (2) whether MRP was a medicines reconciliation discrepancy, as evidence suggests that patients are at increased risk of medication-related harm during transitions of care. ${ }^{9} 32$

MRP data were manually inputted into a spreadsheet by the principal investigator, who performed on-going random checks of approximately $10 \%$ of forms to ensure accurate data entry. Each potential MRP was then independently assessed by an expert panel comprising the principal investigator, a hospital pharmacist, a senior nurse and a consultant physician. The panel validated each MRP through consensus agreement on whether it was a true MRP (expressed as a dichotomous variable of yes or no). Confirmed MRPs that were considered to be preventable were then assessed for severity using a visual analogue scale. ${ }^{33}$ This has 
1 been described in detail elsewhere, with examples of MRPs classified by severity and

2 preventability. ${ }^{13}$

3 An amended version of the aggregated classification system developed by Basger et a/ ${ }^{34}$

4 (see Table 2) was used to categorise the clinically relevant (moderate or severe preventable)

5 MRPs. This was chosen as it is based on the most commonly used classification systems,

6 and provides comprehensive classification based on the causes of MRPs, thereby

7 preventing potential confusion between causes and outcomes. ${ }^{34}$ Three of Basger's MRP

8 subcategories were not used for the present study as they relate only to primary care:

9 'dosage instructions unclear, incomplete or not understood by patient/carer', 'adequate information not provided or not understood or misunderstood or not followed', and 'patient unable to attend/pay for monitoring'. An additional category 'inappropriate abrupt withdrawal of a medicine' was added as this was not captured by Basger's system.

Details of high-risk medicines involved in the clinically relevant MRPs has been published previously. ${ }^{30}$ This gives the impact of groups of high-risk medicines on the risk of developing clinically relevant MRPs, and explores potential correlation between high-risk medicines and other risk factors such as age and renal function.

\section{Data analysis}

Descriptive analyses were performed to identify when clinically relevant MRPs occurred in terms of the stage of inpatient stay, the percentage that were medicine reconciliation discrepancies, and the percentage in each MRP subcategory.

Chi-square tests were performed to test for differences among the stages of inpatient stay in which clinically relevant MRPs were identified for each MRP subcategory. The Bonferroni correction, based on the number of comparisons, was applied to the probability $(p)$ values to account for the risk of type I errors associated with multiple analyses. ${ }^{35}$

Results are reported according to the Strengthening the Reporting of Observational Studies in Epidemiology reporting guidelines for observational studies. ${ }^{36}$ All analyses were conducted using Stata version 14.2.

\section{RESULTS}

An overview of the 1,503 included patients has been presented elsewhere. ${ }^{13}$

\section{MRP descriptive data}

31 A total of 2,736 MRPs were reported for the 1,503 study admissions, 122 (4.5\%) of which

32 were not considered to be true MRPs by the expert panel. 'Unnecessary pharmacy 
contribution', such as advice to use once daily (modified release) oral nitrates rather than twice daily when both were considered to be clinically acceptable, formed the largest category, accounting for $50(41 \%)$ of non-validated MRPs. The second largest category was non-clinically significant drug interactions, accounting for 29 (24\%). Of the 2,614 MRPs considered by the expert panel to be true MRPs, 1,153 were rated as both moderate or severe, and preventable.

Descriptive data for these clinically relevant MRPs are summarised in Table 1. This shows that clinically relevant MRPs were more frequently identified during/before the first wardbased pharmacy review of patients (73.9\% of all clinically relevant MRPs). In total, $52.4 \%$ of clinically relevant MRPs were related to medicines reconciliation discrepancies.

The classification of clinically relevant MRPs is summarised in Table 2; the most frequently identified subcategory was 'indication not treated/missing therapy', accounting for $45.9 \%$ of clinically relevant MRPs. Dose selection issues were the next most frequently reported, with 'dose too low' and 'dose too high' accounting for $13.2 \%$ and $10.8 \%$ of clinically relevant MRPs respectively.

\section{MRP subcategories}

Differences among the stages of hospital stay during which different subcategories of clinically relevant MRPs were identified are summarised in Table 2. Given the Bonferroni corrected $p$ value of 0.002 , there was evidence for differences in the stage during which clinically relevant MRPs were identified for five MRP subcategories: indication not treated/missing therapy $(p<0.001)$, duration of treatment too long $(p<0.001)$, drug underused/under-administered $(p<0.001)$, drug not taken/administered at all $(p<0.001)$, and prescribed drug not available $(p<0.001)$. For the subcategory 'indication not treated $/ \mathrm{missing}$ therapy', identification was more frequent at admission and discharge $(52.7 \%$ and $45.1 \%$ of MRPs identified at each stage respectively) compared to during the inpatient stay (13.6\%). For the remaining four subcategories, the highest percentages were identified during the remainder of inpatient stay.

\section{DISCUSSION}

Clinically relevant MRPs were more frequently identified during/before the first ward-based pharmacy review of patients (73.9\% of all clinically relevant MRPs).The most frequently identified MRP subcategories were 'indication not treated/missing therapy' and medication dosing issues, accounting for almost $70 \%$ of clinically relevant MRPs. For the subcategory 'indication not treated/missing therapy', identification was more frequent at admission and 
1 discharge (52.7\% and $45.1 \%$ of MRPs identified at each stage respectively) compared to 2 during admission (13.6\%). Clinically relevant MRPs related to dosing issues appear to occur 3 more frequently during the remainder of inpatient stay, although this finding did not reach

4 statistical significance.

5 Strengths of this research include prospective data collection and inclusion of consecutive 6 admissions, this enabled optimal measurement of MRPs, and minimised sampling bias.

7 MRP identification by pharmacy staff was also a strength as it (1) permitted identification of

8 MRPs that are not routinely recorded in medical records, such as potential prescribing or administration errors that are intercepted and rectified; and (2) meant MRPs were identified by staff personally involved in the care of the study patients, increasing clinical and practical relevance. Other strengths include the relatively large sample size, and use of two study sites to increase generalisability.

A potential limitation was the possibility of incomplete data due to the observational nature of the study, and pharmacy staff being required to complete this work in addition to other routine duties. To minimise this we worked with the study sites to ensure data collection occurred during an optimal period, and providing training for all pharmacists involved in the study to improve the consistency and reliability of data collection. Other possible limitations were that the expert panel had no access to medical records when severity rating MRPs, which may have led to misclassification, as well as the simple descriptive nature of the analysis, which does not address possible confounding such as experience/grade of pharmacy staff. The results should therefore be interpreted with caution.

In terms of the stage during hospital stay when MRPs occur, we are not aware of previous research that focuses specifically on clinically relevant MRPs. However, a number of studies have investigated the prevalence of prescribing errors, a significant subset of MRPs, ${ }^{37}$ throughout hospital stay. ${ }^{26-28}$ While it is not possible to directly compare these results to the present study due to differences in the outcome measure, data collection methods and analyses used, our findings appear to be consistent; Tully et $a^{26}$ and Franklin et a/27 found that prescribing errors were more likely at admission than at other times. Similarly Ashcroft et $a^{28}$ found that both prescribing errors and 'significant' prescribing errors were more likely to occur at the time of hospital admission when compared to during hospital stay.

Regarding the distribution of MRP subcategories, it is not possible to directly compare results between the present and previously published studies due to the use of different MRP classification systems, ${ }^{7} 3839$ severity rating, and/or outcome measures. ${ }^{27} 28$ However, there are similarities between our findings and previous research; we identified 'indication not treated/missing therapy' as the highest MRP subcategory, accounting for $45.9 \%$ of all 
1 clinically relevant MRPs, which is comparable with Wilmer et al, ${ }^{39}$ where 'under-treatment' accounted for $35.5 \%$ of MRPs (severity not assessed). Wilmer et al also reported that incorrect dosing (overdose or under-dose) accounted for $25 \%$ of MRPs, which is similar to the $24 \%$ found in the present study. Similarly, Franklin et $a^{27}$ found that omission of clinically indicated medication and incorrect dose where the two most commonly identified prescribing error types. Ashcroft et $a^{28}$ also found that omission of required therapy at the time of hospital admission occurred almost three times more frequently than any other prescribing error, accounting for $28.5 \%$ of all errors; under-dosing and over-dosing of medication were the next most common, accounting for $10.9 \%$ and $8.4 \%$ respectively.

Analyses of the stage during hospital stay when clinically relevant MRPs were identified found that over $70 \%$ were recorded during/before the first ward-based review by a pharmacist. Future research may be warranted to investigate whether this was influenced by working practices at the study sites, as it may reflect a focus on newly admitted patients. input, in terms of identification and resolution of clinically relevant MRPs, during the early stages of their admission. Regarding subsequent stages in hospital stay, $15.3 \%$ of clinically relevant MRPs occurred during the 'remainder of inpatient stay', with $10.6 \%$ occurring during clinical screening of discharge prescriptions; this suggests the occurrence of clinically relevant MRPs may diminish throughout the hospital stay, but that patients continue to require pharmacy review.

While prioritisation based on the stage of hospital stay may offer opportunities to increase the efficiency of pharmacy services, it is possible that use of a clinical prioritisation tool may add additional benefits. ${ }^{13}$ Given the high prevalence of clinically relevant MRPs at admission to hospital, a prioritisation tool could be used on hospital admission to determine the level of review required. This could indicate if medicines reconciliation/structured medication review is required, and/or be used to allocate team members appropriately based on their knowledge, skills and expertise. Subsequent prioritisation decisions could then be guided by professional judgement, or prioritisation scores recalculated if there is a significant change in risk, for example due to the initiation of high-risk medicines, resulting in escalation/deescalation as appropriate. Use of this type of combined targeting could permit deprioritisation of lower risk patients, releasing pharmacists' capacity to focus on those patients in greatest need of their input. It may also permit more efficient use of skill mix. For example, input to low-risk patients could initially be limited to simple face-to-face discussions, led by pharmacy technicians, to screen for issues relating to medicines adherence, medicationrelated support needs and/or the drug use process. As we have suggested previously, ${ }^{13}$ other triggers for pharmacy review could also be used, such as swallowing difficulties and 
1 end of life care. This would provide pharmacy teams with a suite of tools, permitting effective

2 prioritisation and allocation of tasks.

3 Regarding the high percentage of clinically relevant MRPs categorised as 'indication not

4 treated/missing therapy' at hospital admission (52.7\%), this may be related to the

5 errors/unintentional changes to medicines that are known to occur on transitions between

6 care settings. ${ }^{3}$ This is supported by our finding that over half of all clinically relevant MRPs

7 were related to medicines reconciliation discrepancies, a process often undertaking during

8 the first pharmacy review. ${ }^{3}$ We also found the category 'indication not treated/missing

9 therapy' formed a high percentage of clinically relevant MRPs at hospital discharge (45.1\%),

10 which is consistent with previous findings that medication errors, often due to omission of a medication, are common on hospital discharge prescriptions. ${ }^{40}$ The results of the present study therefore support recommendations from previous studies, which call for focused pharmacist input at admission and discharge to perform medicines reconciliation. ${ }^{26} 2840$ Although not reaching statistical significance, the category 'no indication/duplication' was more frequently identified at discharge compared to other stages. While one may expect this category of MRP to be resolved during inpatient stay, the majority related to errors introduced when discharge prescriptions were written.

The analysis of MRP subcategories identified during the 'remainder of inpatient stay' suggests that the types/categories of clinically relevant MRPs were more varied compared with those at admission and discharge; prevalence was spread more evenly across the following subcategories: drug selection, dosing, duration, omissions and logistical issues. This may suggest that during the remainder of the inpatient stay, pharmacy services may need to provide ongoing clinical assessment, together with services to address practical/procedural issues related to medicines supply and administration such as excessive duration of use, incorrect/incomplete prescriptions, dose omissions and lack of drug availability.

\section{CONCLUSIONS}

By focussing on clinically relevant MRPs, this study provides insight into the stages of hospital stay during which risk of medication-related patient harm is greatest. This has potential to permit pharmacists to target patients, improving productivity, efficiency and patient safety. We found that patients are at greatest need of pharmacy input, in terms of identification and resolution of clinically relevant MRPs, during the early stages of their inpatient stay. Our results also support the need for medicines reconciliation at admission and discharge, and suggest that during the remainder of the inpatient stay there is also need 
1 for ongoing clinical pharmacy review, alongside services to address practical/procedural

2 issues related to medicines supply and administration.

3 DECLARATIONS

\section{Conflict of interests}

5 The authors declare that they have no conflicts of interests to disclose. 


\section{Funding}

2 This work was supported by a Clinical Doctoral Research Fellowship award from Health

3 Education England (HEE) and the National Institute for Health Research (NIHR), CDRF-

4 2014-05-033. This article represents independent research supported by the

5 NIHR Imperial Patient Safety Translational Research Centre and the NIHR Health Protection

6 Research Unit in Healthcare Associated Infections and Antimicrobial Resistance at Imperial

7 College in partnership with Public Health England (PHE).

8 Disclaimer: The views expressed are those of the author(s) and not necessarily those of the 9 NHS, the NIHR, PHE or the Department of Health and Social Care. The funder had no role 10 in study design, data collection and analysis, decision to publish, or preparation of the 11 manuscript. 
1. The Royal Pharmaceutical Society. SHAPING PHARMACY FOR THE FUTURE Hospital Pharmacy: A briefing for members in England, 2017.

2. Lord Carter of Coles. Operational productivity and performance in English NHS acute hospitals: Unwarranted variations, 2016.

3. National Institute for Health and Care Excellence. Medicines optimisation: the safe and effective use of medicines to enable the best possible outcomes, NICE guidelines [NG5], 2015.

4. NHS England. NHS Long Term Plan, 2019.

5. Krähenbühl-Melcher A et al. Drug-Related Problems in Hospitals. Drug Saf 2007;30(5):379-407. doi: 10.2165/00002018-200730050-00003

6 . Roughead EE et al. The extent of medication errors and adverse drug reactions throughout the patient journey in acute care in Australia. International journal of evidence-based healthcare 2016;14(3-4):113-22.

7. Blix HS et al. The majority of hospitalised patients have drug-related problems: results from a prospective study in general hospitals. Eur J Clin Pharmacol 2004;60(9):65158.

8. van den Bemt PMLA et al. Drug-Related Problems in Hospitalised Patients. Drug Saf 2000;22(4):321-33. doi: 10.2165/00002018-200022040-00005

9. World Health Organization. WHO Global Patient Safety Challenge: Medication Without Harm, 2017.

10. The Society of Hospital Pharmacists of Australia. Standards of Practice for Clinical Pharmacy Services, 2016.

11. Health Quality and Safety Commission New Zealand. All hands on deck: prioritisation criteria 2011 [Available from: https://www.hqsc.govt.nz/assets/MedicationSafety/Med-Rec-PR/MR-Workshop-2011/MR-Workshop-All-hands-on-deckPrioritisation-criteria-Nirasha-Parsotam.pdf accessed June 2019.

12. American Society of Health-System Pharmacists. The consensus of the Pharmacy Practice Model Summit. Am J Health Syst Pharm 2011;68:1148-52.

13. Geeson $C$ et al. Development and performance evaluation of the Medicines Optimisation Assessment Tool (MOAT): a prognostic model to target hospital pharmacists' input to prevent medication-related problems. BMJ Qual Saf 2019:bmjqs-2018-008335.

14. Urbina $O$ et al. Design of a score to identify hospitalized patients at risk of drug-related problems. Pharmacoepidemiology and drug safety 2014;23(9):923-32.

15. Onder $\mathrm{G}$ et al. Development and validation of a score to assess risk of adverse drug reactions among in-hospital patients 65 years or older: the GerontoNet ADR risk score. Arch Intern Med 2010;170(13):1142-48.

16. Tangiisuran $B$ et al. Development and validation of a risk model for predicting adverse drug reactions in older people during hospital stay: Brighton Adverse Drug Reactions Risk (BADRI) model. PloS one 2014;9(10):e111254.

17. Kiguba $R$ et al. Incidence, risk factors and risk prediction of hospital-acquired suspected adverse drug reactions: a prospective cohort of Ugandan inpatients. BMJ Open 2017;7(1) doi: 10.1136/bmjopen-2015-010568

18. McElnay J et al. Development of a risk model for adverse drug events in the elderly. Clinical drug investigation 1997;13(1):47-55.

19. Trivalle $C$ et al. Risk factors for adverse drug events in hospitalized elderly patients: a geriatric score. European Geriatric Medicine 2011;2(5):284-89.

20. Nguyen T-L et al. Improving medication safety: Development and impact of a multivariate model-based strategy to target high-risk patients. PloS one 2017;12(2):e0171995.

21. Cottrell $R$ et al. Developing and implementing a pharmacy risk screening tool. Hospital Pharmacy Europe 2013(71):58-60. 
22. Falconer $\mathrm{N}$ et al. Development of an electronic patient prioritization tool for clinical pharmacist interventions. Am J Health Syst Pharm 2014;71(4):311-20. doi: 10.2146/ajhp130247

23. Roten I et al. Electronic screening of medical records to detect inpatients at risk of drugrelated problems. Pharm World Sci 2009;32(1):103. doi: 10.1007/s11096-009-9352-6

24. Hickson RP et al. Evaluation of a pharmaceutical assessment screening tool to measure patient acuity and prioritise pharmaceutical care in a UK hospital. European Journal of Hospital Pharmacy 2016 doi: 10.1136/ejhpharm-2015-000829

25. Saedder EA et al. Detection of patients at high risk of medication errors: development and validation of an algorithm. Basic \& clinical pharmacology \& toxicology 2016;118(2):143-49.

26. Tully MP, Buchan IE. Prescribing errors during hospital inpatient care: factors influencing identification by pharmacists. Pharm World Sci 2009;31(6):682.

27. Franklin BD et al. Prescribing errors in hospital inpatients: a three-centre study of their prevalence, types and causes. Postgrad Med J 2011;87(1033):739-45.

28. Ashcroft DM et al. Prevalence, nature, severity and risk factors for prescribing errors in hospital inpatients: prospective study in 20 UK hospitals. Drug Saf 2015;38(9):83343.

29. Geeson C et al. Medicines Optimisation Assessment Tool (MOAT): a prognostic model to target hospital pharmacists' input to improve patient outcomes. Protocol for an observational study. BMJ Open 2017;7(6) doi: 10.1136/bmjopen-2017-017509

30. Geeson $C$ et al. High-risk medicines associated with clinically relevant medicationrelated problems in United Kingdom hospitals: a prospective observational study. $\mathrm{Br}$ J Clin Pharmacol 2019;0(ja) doi: 10.1111/bcp.14119

31. Pharmaceutical Care Network Europe. The PCNE Classification V 7.02016 [Available from: http://www.pcne.org/upload/files/152 PCNE classification V7-0.pdf accessed June 2019.

32. The Royal Pharmaceutical Society. Keeping patients safe when they transfer between care providers - getting the medicines right, 2012.

33. Dean BS, Barber ND. A validated, reliable method of scoring the severity of medication errors. Am J Health Syst Pharm 1999;56(1):57-62.

34. Basger BJ et al. Development of an aggregated system for classifying causes of drugrelated problems. Ann Pharmacother 2015;49(4):405-18.

35. Armstrong RA. When to use the Bonferroni correction. Ophthalmic and Physiological Optics 2014;34(5):502-08.

36. Vandenbroucke JP et al. Strengthening the Reporting of Observational Studies in Epidemiology (STROBE): explanation and elaboration. PLoS Med 2007;4(10):e297.

37. Lassetter JH, Warnick ML. Medical errors, drug-related problems, and medication errors: a literature review on quality of care and cost issues. J Nurs Care Qual 2003;18(3):175-83.

38. Ayalew MB et al. Drug-related problems in medical wards of Tikur Anbessa specialized hospital, Ethiopia. Journal of research in pharmacy practice 2015;4(4):216.

39. Wilmer CM et al. Drug-related problems in a clinical setting: a literature review and cross-sectional study evaluating factors to identify patients at risk. Europeon Journal of Hospital Pharmacy 2015;22(4):229-35.

40. Tong EY et al. Reducing medication errors in hospital discharge summaries: a randomised controlled trial. Med J Aust 2017;206(1):36-39. 
1 Table 1 - Descriptive data for 'moderate or severe preventable' medication-related

2 problems (MRPs)

\begin{tabular}{|c|c|}
\hline & $\begin{array}{c}\text { Moderate or } \\
\text { severe } \\
\text { preventable } \\
\text { MRPs = 1,153 } \\
n(\%)\end{array}$ \\
\hline \multicolumn{2}{|l|}{ Stage during patient admission when identified: } \\
\hline During first ward review by pharmacist (or before) & $852(73.9)$ \\
\hline Remainder of inpatient stay & $176(15.3)$ \\
\hline Clinical screening at discharge & $122(10.6)$ \\
\hline Missing data & $3(0.3)$ \\
\hline Medicines reconciliation discrepancy & $604(52.4)$ \\
\hline TOTAL number of moderate or severe preventable MRPs & $1,153(100)$ \\
\hline
\end{tabular}


Table 2 - Classification of 'moderate or severe preventable' medication-related problems

\begin{tabular}{|c|c|c|c|c|c|}
\hline \multirow{3}{*}{ Medication-related problem (MRP) subcategory* } & \multicolumn{3}{|c|}{$\begin{array}{l}\text { Stage during hospital stay when 'moderate or severe } \\
\text { preventable' MRPs identified }\end{array}$} & \multirow{3}{*}{$\begin{array}{c}\text { Total } \\
n=1,153 \S \\
n(\%)\end{array}$} & \multirow{3}{*}{$\begin{array}{c}\text { p value } \\
\text { (test for } \\
\text { difference } \\
\text { among stages } \\
\text { of admission) }\end{array}$} \\
\hline & $\begin{array}{l}\text { During first ward } \\
\text { review (or before) }\end{array}$ & $\begin{array}{l}\text { Remainder of } \\
\text { inpatient stay }\end{array}$ & $\begin{array}{l}\text { Clinical screening } \\
\text { at discharge }\end{array}$ & & \\
\hline & $\begin{array}{c}n=852 \\
n(\%)\end{array}$ & $\begin{array}{l}n=176 \\
n(\%)\end{array}$ & $\begin{array}{c}n=122 \\
n(\%)\end{array}$ & & \\
\hline \multicolumn{6}{|l|}{ 1. Drug selection } \\
\hline 1.1 Inappropriate drug & $45(5.3)$ & $13(7.4)$ & $5(4.1)$ & $63(5.5)$ & 0.417 \\
\hline 1.2 No indication for drug/duplication & $18(2.1)$ & $6(3.4)$ & $8(6.6)$ & $32(2.8)$ & 0.017 \\
\hline 1.3 Interaction (drug-drug, or drugs and food/alcohol & $22(2.6)$ & $1(0.6)$ & $2(1.6)$ & $25(2.2)$ & 0.227 \\
\hline 1.4 Indication not treated/missing therapy & $449(52.7)$ & $24(13.6)$ & $55(45.1)$ & $529(45.9)$ & $<0.001$ \\
\hline 1.5 More cost effective drug available & 0 & 0 & 0 & 0 & $\mathrm{~N} / \mathrm{A}$ \\
\hline 1.6 Synergistic/preventive drug required and not given & $4(0.5)$ & 0 & 0 & $4(0.4)$ & 0.496 \\
\hline \multicolumn{6}{|l|}{ 2. Drug form } \\
\hline 2.1 Inappropriate or suboptimal drug form & $9(1.1)$ & $1(0.6)$ & $3(2.5)$ & $13(1.1)$ & 0.291 \\
\hline \multicolumn{6}{|l|}{ 3. Dose selection } \\
\hline 3.1 Drug dose too low & $111(13)$ & $29(16.5)$ & $12(9.8)$ & $152(13.2)$ & 0.238 \\
\hline 3.2 Drug dose too high & $78(9.2)$ & $31(17.6)$ & $15(12.3)$ & $124(10.8)$ & 0.004 \\
\hline 3.3 Dosage regimen not frequent enough & $2(0.2)$ & 0 & 0 & $2(0.2)$ & 0.704 \\
\hline 3.4 Dosage regimen too frequent & $4(0.5)$ & $1(0.6)$ & $1(0.8)$ & $6(0.5)$ & 0.878 \\
\hline $\begin{array}{l}\text { 3.5 Dose needs adjustment to organ function or change in } \\
\text { disease state }\end{array}$ & $17(2.0)$ & $7(4.0)$ & $1(0.8)$ & $25(2.2)$ & 0.144 \\
\hline $\begin{array}{l}\text { 3.6 Dosage instructions unclear, incomplete or not } \\
\text { understood by patient/carer }{ }^{\dagger}\end{array}$ & $\mathrm{N} / \mathrm{A}$ & $\mathrm{N} / \mathrm{A}$ & N/A & $\mathrm{N} / \mathrm{A}$ & $\mathrm{N} / \mathrm{A}$ \\
\hline \multicolumn{6}{|l|}{ 4. Treatment duration/withdrawal } \\
\hline 4.1 Duration of treatment too short & $1(0.1)$ & 0 & $1(0.8)$ & $2(0.2)$ & 0.183 \\
\hline 4.2 Duration of treatment too long & $5(0.6)$ & $9(5.1)$ & $3(2.5)$ & $17(1.5)$ & $<0.001$ \\
\hline 4.3 Inappropriate abrupt withdrawal ${ }^{\ddagger}$ & $2(0.2)$ & 0 & 0 & $2(0.2)$ & 0.704 \\
\hline
\end{tabular}




\begin{tabular}{|c|c|c|c|c|c|}
\hline \multicolumn{6}{|l|}{ 5. Drug use process } \\
\hline $\begin{array}{l}5.1 \text { Inappropriate timing of administration/dosing by } \\
\text { prescriber; administration error by nurse }\end{array}$ & $6(0.7)$ & $4(2.3)$ & $1(0.8)$ & $11(1.0)$ & 0.148 \\
\hline 5.2 Drug underused/under-administered & $6(0.7)$ & $10(5.7)$ & $1(0.8)$ & $17(1.5)$ & $<0.001$ \\
\hline 5.4 Drug not taken/administered at all & $4(0.5)$ & $7(4.0)$ & 0 & $11(1.0)$ & $<0.001$ \\
\hline 5.5 Wrong drug taken by patient & 0 & 0 & 0 & 0 & N/A \\
\hline 5.6 Drug abused & 0 & 0 & 0 & 0 & $N / A$ \\
\hline $\begin{array}{l}\text { 5.8 Adequate information not provided or not understood or } \\
\text { misunderstood or not followed }^{\dagger}\end{array}$ & $\mathrm{N} / \mathrm{A}$ & N/A & $\mathrm{N} / \mathrm{A}$ & $\mathrm{N} / \mathrm{A}$ & N/A \\
\hline $\begin{array}{l}5.9 \text { Drugs stored inappropriately/expired drug } \\
\text { administered/preparation error }\end{array}$ & & 0 & 0 & $3(0.3)$ & 0.591 \\
\hline \multicolumn{6}{|l|}{ 6. Logistics } \\
\hline 6.1 Prescribed drug not available & $2(0.2)$ & $12(6.8)$ & 0 & $16(1.4)$ & $<0.001$ \\
\hline 7.1 Monitoring too frequent & 0 & 0 & 0 & 0 & N/A \\
\hline 7.2 No or too infrequent monitoring & $1(0.1)$ & $2(1.1)$ & 0 & $3(0.3)$ & 0.046 \\
\hline 7.3 Inappropriate test ordered & 0 & 0 & 0 & 0 & N/A \\
\hline 7.4 Patient unable to attend/pay for monitoring ${ }^{\dagger}$ & N/A & N/A & N/A & $\mathrm{N} / \mathrm{A}$ & N/A \\
\hline \multicolumn{6}{|c|}{ 8. Unexpected reaction/adverse drug reaction (ADR) / no obvious cause } \\
\hline 8.1 An ADR occurred & 0 & $1(0.6)$ & 0 & $1(0.1)$ & 0.063 \\
\hline 8.2 No obvious cause of treatment failure & 0 & 0 & 0 & 0 & N/A \\
\hline TOTAL number of moderate or severe preventable MRPs & $852(100)$ & $176(100)$ & $122(100)$ & $1,153(100)$ & N/A \\
\hline
\end{tabular}


$1 \dagger \quad$ Category not used for present study as relates only to primary care.

$2 \quad$ Category not included in Basger's original classification system.

$3 \S \quad$ Data on 'stage during admission' when moderate or severe preventable MRP identified missing for three MRPs.

$4 \quad \mathrm{~N} / \mathrm{A}=$ not applicable.

5 Bonferroni adjusted $p$ value used to judge statistical significance 0.002 (based on 23 statistical tests). 


\section{Point by point response to the reviewer comments}

\begin{tabular}{|c|c|}
\hline Reviewer comment & Author's response \\
\hline \multicolumn{2}{|l|}{ Associate Editor Comments to Author: } \\
\hline \multirow{3}{*}{$\begin{array}{l}\text { 1. Justify the data collection period? Sorry } \\
\text { I did not notice this before- was there an a } \\
\text { priori power calculation? }\end{array}$} & Thank you for the opportunity to expand on this point. \\
\hline & $\begin{array}{l}\text { The sample size was determined a priori based on practical } \\
\text { considerations including funding, time available, and } \\
\text { accessibility of data at the study sites. While sample size is } \\
\text { often calculated based on power calculations, this was not } \\
\text { possible as there was not a clear 'measure of effect' to } \\
\text { power the research. }\end{array}$ \\
\hline & $\begin{array}{l}\text { A brief summary of this information has now been added to } \\
\text { the method section of the main manuscript (page } 3 \text {, line } 29 \text { - } \\
30 \text { ). }\end{array}$ \\
\hline \multicolumn{2}{|l|}{ 2. Coreect the following typos: } \\
\hline $\begin{array}{l}\text { Page } 3 \text { line } 27 \text { missing 'were' between } \\
\text { charts and rewritten }\end{array}$ & Amended as advised. \\
\hline $\begin{array}{l}\text { Page } 8 \text { line } 4 \text { under doing should be under } \\
\text { dosing? }\end{array}$ & $\begin{array}{l}\text { Thank you for identifying this error. It has been amended as } \\
\text { advised. }\end{array}$ \\
\hline $\begin{array}{l}\text { Page } 8 / 9 \text { could you Nebraskan's up this } \\
\text { long paragraph? }\end{array}$ & $\begin{array}{l}\text { We assume that you are suggesting that we break up this } \\
\text { paragraph. We have therefore now broken this paragraph } \\
\text { into shorter sections. }\end{array}$ \\
\hline
\end{tabular}

